\title{
Time and Dose-Dependent Effects of Viscum Album Quercus on Rabbit Spermatozoa Motility and Viability in Vitro
}

\author{
M. HALO Jr. ${ }^{1}$, P. MASSANYI ${ }^{1,2}$, A. GREN ${ }^{2}$, A. LASAK $^{2}$, T. SLANINA ${ }^{1}$, L. ONDRUSKA ${ }^{3}$, \\ R. MUCHACKA ${ }^{2}$, D. GALBAVY ${ }^{4}$, P. IVANIC ${ }^{5}$, E. R. SCHNEIR ${ }^{6}$, G. FORMICKI ${ }^{2}$ \\ ${ }^{1}$ Department of Animal Physiology, Faculty of Biotechnology and Food Sciences, Slovak \\ University of Agriculture, Nitra, Slovak Republic, ${ }^{2}$ Department of Animal Physiology and \\ Toxicology, Institute of Biology, Faculty of Geography and Biology, Pedagogical University of \\ Cracow, Cracow, Poland, ${ }^{3}$ Institute of Farm Animals, Animal Production Research Centre Nitra, \\ Luzianky, Slovak Republic, ${ }^{4}$ Avelane Clinic, Nitra, Slovak Republic, ${ }^{5}$ Slovak Biological Services, \\ Banska Bystrica, Slovak Republic, ${ }^{6}$ Faculty of Planning, The National University Agraria La \\ Molina, Lima, Peru
}

Received May 31, 2019

Accepted July 31, 2019

Epub Ahead of Print October 25, 2019

\section{Summary}

The target of this study was to evaluate the effect of extract of the European mistletoe - Viscum album quercus L. on spermatozoa motility and viability in vitro. The CASA system was used to determine the spermatozoa motility parameters at different time intervals $(0,1,2$ and $3 \mathrm{~h})$ and spermatozoa viability was determined in five different doses of Viscum album quercus $\mathrm{L}[10(\mathrm{QA}), 6.6(\mathrm{QB}), 3.3(\mathrm{QC}), 2.5(\mathrm{QD})$ and $2(\mathrm{QE})$ $\mathrm{mg} / \mathrm{ml}$. Results in experimental groups detected a significant deterioration on rabbit spermatozoa after 1, 2 and 3 hours, compared to the control. The initial total spermatozoa motility showed increased value for all doses of Viscum album quercus in comparison to control. After in vitro culture a dose-dependent decrease (QA: reduction of $69.7 \%, \mathrm{QB}$ : reduction of $40.9 \%$ ) was found. For the progressive spermatozoa most significant decrease (86.8\% for QA vs. $48.5 \%$ for $\mathrm{QB}$ ) was detected compared to the control after 3 hours of culture. Spermatozoa viability (MTT test) was decreased in all experiment groups at the end of experiment, but the differences were not significant. Significant alterations of membrane integrity were found in groups with the highest Viscum album quercus concentration (QA, QB), but acrosome integrity showed no significant changes. Results suggest negative dose- and time-dependent effect of Viscum album quercus at higher doses on spermatozoa motility and viability parameters in vitro.
\end{abstract}

\section{Key words}

Viscum album • Spermatozoa • CASA • Viability • in vitro

\section{Corresponding author}

M. Halo, Department of Animal Physiology, Faculty of Biotechnology and Food Sciences, Slovak University of Agriculture, Trieda Andreja Hlinku 2, 94976 Nitra, Slovak Republic. E-mail: markohalo@yahoo.com

\section{Introduction}

Anticancer preparations made from plants have been an object of scientific interest for many years. It is worth noting that as many as $25 \%$ of cytostatics used in the anticancer chemotherapy are obtained from plants.

Extracts of the European mistletoe (Viscum album L.) have been widely used for decades as alternative, complementary treatment (Kovacs et al. 2006, Felenda et al. 2019, Suveren et al. 2017). In clinical practice mistletoe therapy is often given concomitantly to conventional chemotherapy. Mistletoe plants are generally growing on different host trees, like apple, oak, or pine. Cytotoxic glycoproteins, the mistletoe lectins, are active component of mistletoe extracts and can stimulate effector cells of the innate and adaptive immune system (Stein et al. 2002, Braedel-Ruoff 2010, Gren and Formicki 2013, Gren and Massanyi 2016).

Experiments also indicate a statistically significant increase in albumin fraction level and lymphocyte count. Moreover, decrease of the total protein 
content, protein fractions globulins alpha2, beta, gamma and neutrophil, monocyte count in mouse serum was observed (Gren 2009).

The reproductive ability and the semen quality of animal species can be affected by many environmental sources, as well as age, stress, hormonal status, nutrition and toxins (Mangelsdorf et al. 2003, Lukac et al. 2011, Mousa-Balabel and Mohamed 2011, Fallas-López et al. 2011, Tirpak et al. 2017, Saha et al. 2019). In the many years, following the increased success rate of cancer treatments, great efforts have been made to improve quality of life in survivors, including fertility preservation in young patients (Masopotova et al. 2018). Because of their gonadotoxic effects, chemo- and radiotherapy can temporarily or permanently compromise fertility (Di Bisceglie et al. 2013). Oncological treatments present severe gonadotoxic effects on both germ and Leydig cells. Of note, in a significant percentage of patients (20-50\%) spermatogenesis is impaired even before cancer treatments, probably due to the malignancy itself. The recovery of normal spermatogenesis after treatment may require several years, and mainly depends on various factors - initial spermatozoa count, type and dose of specific oncological treatments and patient age. Disturbance of homeostasis of reproductive system in some diseases can be seen from the decline in physical and chemical parameters of spermatozoa, such as $\mathrm{pH}$, semen volume, concentration, motility, and the percentage of spermatozoa viability. These data justify the increasing efforts in identifying prevention and treatment strategies to preserve reproductive functions in young men with malignancies (Colpi et al. 2004, Maltaris et al. 2006, Vitku et al. 2015, Heráček et al. 2018). The mechanisms underlying the male infertility of Viscum album extracts have not been investigated.

The objective of this in vitro study was to determine the effect of various concentrations of Viscum album quercus during various time periods (0-3 h) on the selected parameters of rabbit spermatozoa motility and viability.

\section{Methods}

Animals, semen samples and in vitro culture

Male rabbits ( $n=10$, New Zealand White) kept under standard conditions at the Experimental Station of the Animal Production Research Centre Nitra, Slovak Republic were selected on the basis of age normally associated with reproduction (12-14 months). Animals were housed in a partially air-conditioned rabbit house (Animal Production Research Centre, Nitra) under a photoperiod 16L : 8D (minimum light intensity of 80 lux). Animals were kept in individual cages and fed with a commercial diet and were provided water ad libitum. An air temperature of $20 \pm 2{ }^{\circ} \mathrm{C}$ and relative humidity of $70 \pm 5 \%$ was maintained in the rabbit house. Conditions of their care, manipulations and use corresponded to the instruction of EC no. 178/2002 and related EC documents, and they were approved by local ethics committee.

Semen samples $(n=5)$ in five replicates were collected on a single day (early in the morning) with the help of artificial vagina (Krockova et al. 2012, Parkanyi et al. 2015). Immediately after collection the individual doses of semen exhibiting a white color without presence of any gel and artificial particles, were mixed together to obtain pooled sample. The spermatozoa concentration in semen was $0.40-0.63 \times 10^{9}$ per $\mathrm{ml}$. The obtained semen samples were diluted according to routine methods (Chrenek et al. 2007, Roychoudhury and Massanyi 2008). Later the spermatozoa were incubated in thermostat $\left(37 \pm 0.5^{\circ} \mathrm{C}\right)$ with various concentrations of Viscum album

Table 1. Concentrations of Viscum album quercus used in the study.

\begin{tabular}{|c|c|c|c|c|}
\hline Group & Semen $(\mu \mathrm{l})$ & $\begin{array}{l}\text { Iscador Qu } \\
10 \mathrm{mg}(\mu \mathrm{l})\end{array}$ & $\begin{array}{c}\text { Physiological } \\
\text { solution }(\mu \mathrm{l})\end{array}$ & $\begin{array}{l}\text { Concentration of } \\
\text { Iscador } \mathbf{Q u} \text { in } \\
\text { samples }(\mathrm{mg} / \mathrm{ml})\end{array}$ \\
\hline$Q K-$ control & 100 & 0 & 300 & 0 \\
\hline$Q A$ & 100 & 300 & 0 & 10 \\
\hline$Q B$ & 100 & 200 & 100 & 6.6 \\
\hline$Q C$ & 100 & 100 & 200 & 3.3 \\
\hline$Q D$ & 100 & 100 & 300 & 2.5 \\
\hline$Q E$ & 100 & 100 & 400 & 2 \\
\hline
\end{tabular}


quercus (Iscador Qu 10 mg, Weleda, Verein für Krebsforschung Institute Hiscia - Arlesheim, Switzer-land) dissolved in physiological solution. Concentrations of the Iscador Qu were based on manufacturer's values and later diluted with physiological solution to reach decreased experimental concentration (6.6-2.0 mg). The control (QK) group was cultured only with physiological solution. The scheme of experiments is presented in Table 1.

\section{Computer-assisted semen analysis}

Spermatozoa motility was used as an indicator of cell quality. The motility analysis was carried out using a CASA (Computer Assisted Semen Analysis) system Sperm Vision TM program (MiniTub, Tiefenbach, Germany) with the Olympus BX 51 microscope (Olympus, Tokyo, Japan) at cultivation times 0, 1, 2 and 3 hours (Time 0-3). Each sample was placed into the Makler Counting Chamber (depth $10 \mu \mathrm{m}$, Sefi-Medical Instruments, Haifa, Israel (Massanyi et al. 2008). This study was performed in five replicates at each concentration. At least 1000 spermatozoa were analyzed in each sample (Lukac et al. 2013, Halo et al. 2018). Using the rabbit specific set up the following parameters have been evaluated - total motility (MOT, \%), progressive motility (PRO,\%), distance average path (DAP, $\mu \mathrm{m}$ ), distance curved line (DCL, $\mu \mathrm{m}$ ), distance straight line (DSL, $\mu \mathrm{m}$ ), average path velocity (VAP, $\mu \mathrm{m} / \mathrm{s}$ ), velocity curved line (VCL, $\mu \mathrm{m} / \mathrm{s}$ ), velocity straight line (VSL, $\mu \mathrm{m} / \mathrm{s}$ ), straightness (STR), linearity (LIN), wobble (WOB), amplitude of lateral head displacement $(\mathrm{ALH}, \mu \mathrm{m})$ and beat-cross frequency $(\mathrm{BCF}, \mathrm{Hz})$ as described previously (Roychoudhury et al. 2010, Tvrda et al. 2015 Adamkovicova et al. 2016).

\section{Viability analysis - MTT test}

Viability of rabbit spermatozoa cultured with Viscum album quercus was evaluated by the metabolic activity (MTT) assay after 3 hours of culture. This colorimetric assay measures the conversion of 3-(4.5dimetylthiazol-2-yl)-2.5-diphenyltetrazolium bromide (MTT, Sigma-Aldrich, St. Louis, USA) to purple formazan particles by mitochondrial succinate dehydrogenase of intact mitochondria of living cells. Formazan was measured spectrophotometrically by a microplate ELISA reader (Multiskan FC, ThermoFisher Scientific, Finland). The data are expressed in percentage of control. Results from the analysis were collected during four repeated experiments for each concentration (Slanina et al. 2016).

\section{Viability - membrane integrity - Eosin-nigrosin}

The spermatozoa viability was evaluated using eosin-nigrosin staining methods (Slanina et al. 2018). From all the samples smears were prepared after 3 hours of culture. Experimental samples and the control sample were diluted in the ratio $1: 2$ : 2 with $5 \%$ eosin (Eosin Y) and 10\% nigrosin (Nigrosin) solution (both Sigma-Aldrich, St. Louis, USA). For each slide 300 cells were counted under a light microscope $(1000 \times$, Leica DMIL LED, Leica Microsystems CMS GmbH, Germany) and classified as viable (intact membrane) and dead (damaged membrane). The experiment was realized in six replicates. The results of viability evaluation were expressed as the percentage of viable and dead spermatozoa (in \%).

\section{Acrosomal integrity}

The acrosomal status was assessed after 3 hours of culture following the fast green-rose Bengal staining protocol designed by Pope et al. (1991). This single-step staining method applies a mixture consisting of $1 \%$ fast green (Sigma-Aldrich, St. Louis, USA), $1 \%$ rose bengal (Sigma-Aldrich, St. Louis, USA) and $40 \%$ ethyl alcohol (Centralchem, Bratislava, Slovak Republic) in $0.1 \mathrm{M}$ citric acid - 0.2 M disodium phosphate buffer (SigmaAldrich, St. Louis, USA). Twenty microliters of the sample were mixed with $20 \mu$ l of the staining solution and incubated for $70 \mathrm{~s}$ at room temperature. Ten microliters of the mixture were smeared on a tempered glass slide and air-dried at $37^{\circ} \mathrm{C}$. Acrosomal integrity was evaluated using bright field microscopy at $1000 \times$ using oil immersion. At least 200 cells per slide were evaluated for the presence or absence of acrosome, and expressed as a percentage rate (Tvrda et al. 2017).

\section{Statistical analysis}

The control group (medium without Viscum album quercus) was compared to the experimental groups. Statistical analysis was carried out using the GraphPad Prism program (version 3.02 for Windows, GraphPad Software, La Jolla California USA). Descriptive statistical characteristics (mean, standard deviation) were evaluated at first. One-way ANOVA with Dunnett's post-test was used for statistical evaluations. The level of significance was set at $* * * \quad(p<0.001)$, $* *(\mathrm{p}<0.01)$ and $*(\mathrm{p}<0.05)$. For individual measurements average value $(\mathrm{x})$, minimum $(\mathrm{min})$ and maximum $(\max )$ value, standard deviation (SD) and coefficient of variation $(\mathrm{CV})$ were recorded. 


\section{Results}

\section{Spermatozoa motility}

The initial spermatozoa motility (Time 0) showed increased value for all doses of Viscum album quercus in comparison to the control group. Statistically significant increase was observed $(p<0.05)$ in the sample QA $(77.23 \pm 8.64 \%)$, and also $(\mathrm{p}<0.001)$ for $\mathrm{QB}$ $(81.17 \pm 8.08 \%)$ and QC $(80.87 \pm 8.83 \%)$. After $1 \mathrm{~h}$ of the culture the average spermatozoa motility in control group was $72.17 \pm 11.94 \%$. At the same time a statistically significant $(\mathrm{p}<0.001)$ decrease $(23.8 \%)$ was observed in group QA $(54.98 \pm 15.53 \%)$. After 2 hours of in vitro cultivation significantly decreased $(\mathrm{p}<0.001$ and 0.01$)$ total spermatozoa motility in comparison to the control group was observed in groups QA and QB (doses 10 and $6.6 \mathrm{mg} / \mathrm{ml}$ ). Also, after 3 hours of in vitro cultivation a dose-dependent decrease (QA: $19.39 \pm 7.7 \%$, reduction of $69.7 \%$, QB: $37.84 \pm 18.29 \%$, reduction of $40.9 \%$ ) was found (Fig. 1).

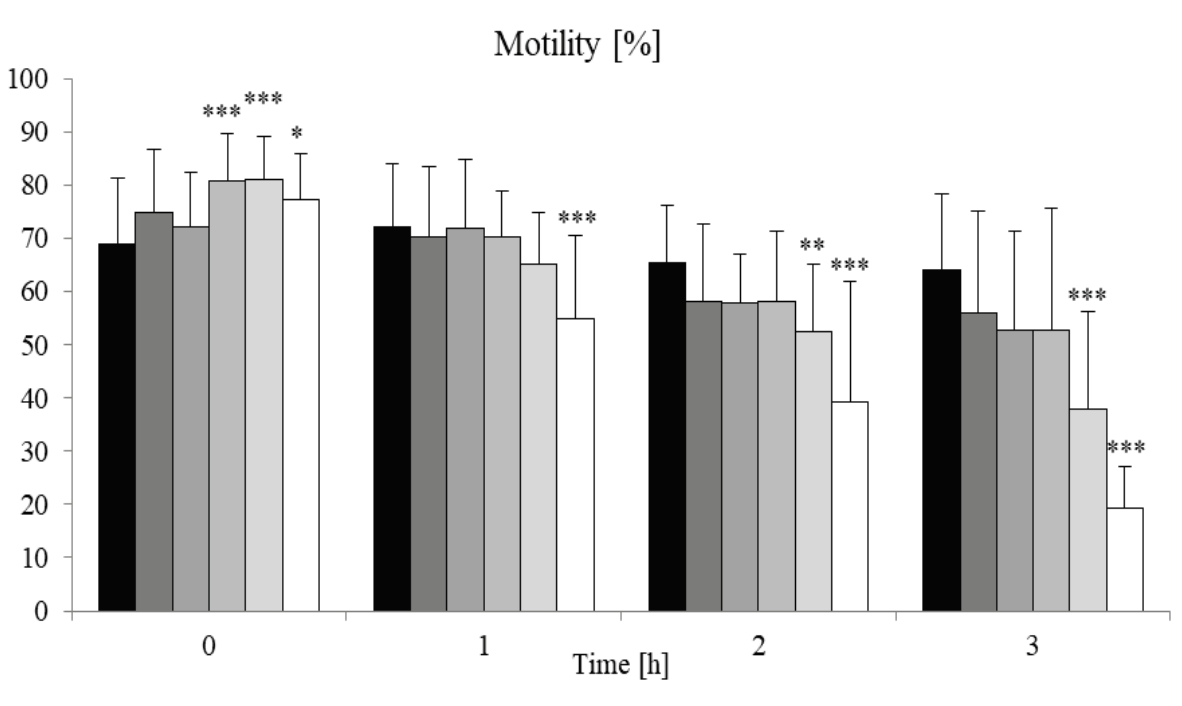

घK $\square \mathrm{QE} \square \mathrm{QD} \backsim \mathrm{QC} \square \mathrm{QB} \square \mathrm{QA}$

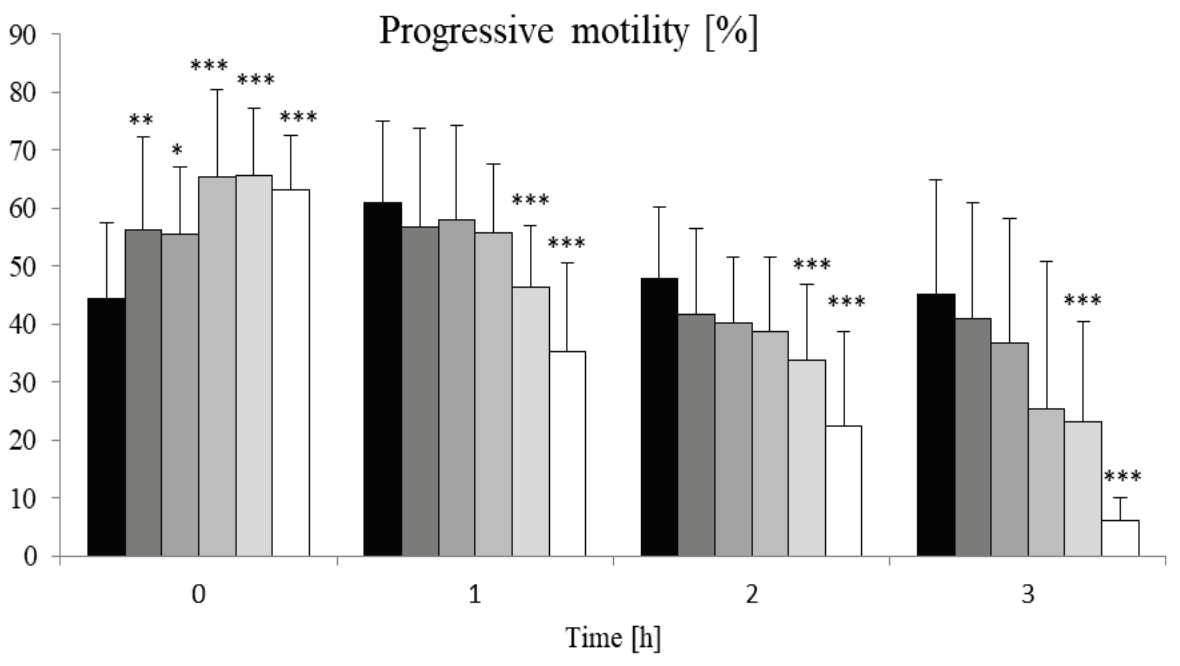

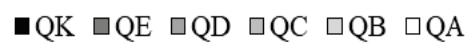

Fig. 1. The effect of Viscum album quercus on the total spermatozoa motility (in \%).

$\mathrm{QK}-0, \mathrm{QE}-2, \mathrm{QD}-2.5, \mathrm{QC}-3.3$, $\mathrm{QB}-6.6, \mathrm{QA}-10 \mathrm{mg} / \mathrm{ml}$ of Iscador $\mathrm{Qu}$. The level of significance was set at $* * *(p<0.001), * *(p<0.01)$ and $*(p<0.05)$.
Fig. 2. The effect of Viscum album quercus on the progressive spermatozoa motility (in \%).

$\mathrm{QK}-0, \mathrm{QE}-2, \mathrm{QD}-2.5, \mathrm{QC}-3.3$, $\mathrm{QB}-6.6, \mathrm{QA}-10 \mathrm{mg} / \mathrm{ml}$ of Iscador $\mathrm{Qu}$. The level of significance was set at $* * *(p<0.001), * *(p<0.01)$ and $*(p<0.05)$.

\section{Progressive spermatozoa motility}

At Time 0 the average progressive spermatozoa motility was higher in all experimental groups with Viscum album quercus in comparison with the control $(44.67 \pm 13.03 \%)$. These changes were statistically significant. After 1 hour of culture the progressive spermatozoa motility was the highest in the control group QK $(60.97 \pm 13.98 \%)$ and a statistically significant decrease was detected in groups QA and QB. This decrease was compared to control $42.4 \%$ for QA and 
$24.2 \%$ for group QB. After 2 hours of in vitro culture, the progressive spermatozoa motility was significantly reduced in groups QA and QB with a decrease up to $53 \%$ in QA $(22.43 \pm 16.34 \%)$ and $29.5 \%$ in group QB (33.63 $\pm 13.31 \%)$. Even more dramatic reduction $(86.8 \%$ for QA vs. $48.5 \%$ for QB) was detected compared to the control (45.12 $\pm 19.61 \%)$ after 3 hours of culture (Fig. 2).

\section{Distance parameters}

Spermatozoa distance path parameters confirm the negative dose and time-dependent effect of Viscum album quercus. Spermatozoa distance average path significantly decreased at Time 1 in experimental groups QA, QB and QC. Later (Time 2) a significant decrease was detected in almost all experimental groups (except the lowest concentration) and at Time 3 only in groups with the highest Viscum album quercus concentrations (Table 2).

Spermatozoa distance curvilinear line showed similar trends as spermatozoa distance path with various significant decrease at all time periods in experimental groups compared to control (Table 3).

Table 2. Spermatozoa distance average path $(\mathrm{DAP}, \mu \mathrm{m})$ in experimental groups and time periods.

\begin{tabular}{|c|c|c|c|c|c|}
\hline Group & Mean & S.D. & C.V. & Minimum & Maximum \\
\hline \multicolumn{6}{|l|}{ Time 0} \\
\hline$Q K$ & 22.90 & 4.43 & 19.36 & 16.02 & 32.75 \\
\hline$Q A$ & 24.56 & 3.79 & 15.43 & 16.82 & 31.60 \\
\hline$Q B$ & 25.69 & 3.92 & 15.25 & 18.35 & 32.61 \\
\hline$Q C$ & 25.41 & 5.21 & 20.52 & 11.97 & 33.70 \\
\hline$Q D$ & 26.14 & 5.76 & 22.02 & 14.05 & 38.53 \\
\hline$Q E$ & 22.97 & 3.99 & 17.37 & 16.40 & 31.49 \\
\hline \multicolumn{6}{|l|}{ Time 1} \\
\hline$Q K$ & 28.91 & 3.69 & 12.75 & 21.19 & 36.69 \\
\hline$Q A$ & $18.05^{* * *}$ & 2.22 & 12.30 & 14.14 & 22.83 \\
\hline$Q B$ & $20.24^{* * *}$ & 2.25 & 11.14 & 16.41 & 26.01 \\
\hline$Q C$ & $22.92^{* * *}$ & 2.38 & 10.38 & 18.70 & 27.44 \\
\hline$Q D$ & 26.23 & 2.42 & 9.21 & 21.15 & 30.61 \\
\hline$Q E$ & 26.49 & 3.44 & 12.99 & 21.54 & 32.76 \\
\hline \multicolumn{6}{|l|}{ Time 2} \\
\hline$Q K$ & 22.51 & 3.34 & 14.84 & 15.29 & 31.10 \\
\hline$Q A$ & $13.27^{* * *}$ & 3.36 & 25.34 & 0.00 & 16.81 \\
\hline$Q B$ & $17.26^{* * *}$ & 2.41 & 13.97 & 12.68 & 21.90 \\
\hline$Q C$ & $17.45^{* * *}$ & 1.88 & 10.80 & 13.81 & 21.56 \\
\hline$Q D$ & $19.02^{* *}$ & 2.72 & 14.30 & 14.45 & 27.30 \\
\hline$Q E$ & 20.55 & 2.80 & 13.60 & 13.64 & 26.45 \\
\hline \multicolumn{6}{|l|}{ Time 3} \\
\hline$Q K$ & 19.54 & 4.31 & 22.06 & 11.80 & 27.42 \\
\hline$Q A$ & $9.40^{* * *}$ & 3.99 & 42.45 & 0.00 & 16.90 \\
\hline$Q B$ & $14.17^{* * *}$ & 4.06 & 28.65 & 0.00 & 22.14 \\
\hline$Q C$ & 16.70 & 4.42 & 26.48 & 8.95 & 22.17 \\
\hline$Q D$ & 18.56 & 4.40 & 23.72 & 11.09 & 27.61 \\
\hline$Q E$ & 18.44 & 3.18 & 17.25 & 12.06 & 22.85 \\
\hline
\end{tabular}

Legend: S.D. - standard deviation, C.V. - coefficient of variation, $* * *(p<0.001), * *(p<0.01)$ (experimental group vs. control). QK -0 , $\mathrm{QE}-2, \mathrm{QD}-2.5, \mathrm{QC}-3.3, \mathrm{QB}-6.6, \mathrm{QA}-10 \mathrm{mg} / \mathrm{ml}$ of Iscador Qu. 
Spermatozoa distance straight line significantly decreased at all time periods in groups QA - QC. Only at Time 2 group QD showed significant decrease (Table 4).

\section{Velocity parameters}

Also the spermatozoa velocity parameters were affected by the addition of Viscum album quercus (Iscador $\mathrm{Qu})$ to the culture medium. The spermatozoa velocity curved line at all time periods in control group was
97.58-131.47 $\mu \mathrm{m} / \mathrm{s}$. After a significant increase at Time 0 (groups QB, QD) a signifacnt decrease was found in all experient groups. Later the most significant decrese was detected in group QA compared to control (131.47 \pm 16.38 vs. $93.25 \pm 10.19$ at Time $1,108.93 \pm 17.72$ vs. $70.77 \pm 19.69$ at Time 2 and $97.58 \pm 21.79$ vs. $47.86 \pm 21.66$ at Time 3). Also in group QB a significant decrease was detected after 1, 2 and 3 hours of culture (Fig. 3).

Table 3. Spermatozoa distance curvilinear line $(\mathrm{DCL}, \mu \mathrm{m})$ in experimental groups and time periods.

\begin{tabular}{|c|c|c|c|c|c|}
\hline Group & Mean & S.D. & C.V. & Minimum & Maximum \\
\hline \multicolumn{6}{|l|}{ Time 0} \\
\hline$Q K$ & 47.84 & 7.80 & 16.30 & 35.09 & 67.59 \\
\hline$Q A$ & 52.25 & 6.81 & 13.03 & 36.55 & 65.73 \\
\hline$Q B$ & 52.76 & 6.52 & 12.35 & 38.31 & 65.55 \\
\hline$Q C$ & 50.01 & 7.19 & 14.37 & 34.42 & 63.38 \\
\hline$Q D$ & $56.03^{* *}$ & 14.35 & 25.61 & 35.85 & 92.81 \\
\hline$Q E$ & 47.66 & 6.48 & 13.59 & 33.89 & 62.09 \\
\hline \multicolumn{6}{|l|}{ Time 1} \\
\hline$Q K$ & 56.01 & 7.39 & 13.19 & 44.27 & 77.16 \\
\hline$Q A$ & $41.19^{* * *}$ & 4.74 & 11.52 & 33.71 & 53.96 \\
\hline$Q B$ & $43.79^{* * *}$ & 4.70 & 10.74 & 36.12 & 53.41 \\
\hline$Q C$ & $45.98^{* * *}$ & 4.07 & 8.86 & 40.28 & 54.68 \\
\hline$Q D$ & 52.54 & 5.33 & 10.14 & 40.52 & 61.28 \\
\hline$Q E$ & 53.61 & 7.27 & 13.56 & 41.29 & 66.05 \\
\hline \multicolumn{6}{|l|}{ Time 2} \\
\hline$Q K$ & 46.87 & 7.61 & 16.24 & 33.81 & 68.31 \\
\hline$Q A$ & $31.38^{* * *}$ & 9.01 & 28.71 & 0.00 & 44.65 \\
\hline$Q B$ & $40.40^{* * *}$ & 4.93 & 12.21 & 31.29 & 50.00 \\
\hline$Q C$ & $38.71^{* * *}$ & 5.07 & 13.09 & 29.61 & 50.94 \\
\hline$Q D$ & $41.62^{*}$ & 5.56 & 13.35 & 28.80 & 59.57 \\
\hline$Q E$ & 45.42 & 5.46 & 12.03 & 31.92 & 58.97 \\
\hline \multicolumn{6}{|l|}{ Time 3} \\
\hline$Q K$ & 42.31 & 9.20 & 21.76 & 27.39 & 60.82 \\
\hline$Q A$ & $20.69^{* * *}$ & 9.53 & 46.07 & 0.00 & 37.80 \\
\hline$Q B$ & $34.22^{* *}$ & 10.26 & 29.97 & 0.00 & 52.86 \\
\hline$Q C$ & 37.83 & 9.40 & 24.85 & 21.49 & 49.89 \\
\hline$Q D$ & 43.07 & 8.81 & 20.45 & 25.50 & 58.38 \\
\hline$Q E$ & 41.58 & 7.61 & 18.31 & 25.81 & 54.31 \\
\hline
\end{tabular}

Legend: S.D. - standard deviation, C.V. - coefficient of variation, $* * *(p<0.001), * *(p<0.01)$ and $*(p<0.05)$ (experimental group vs. control). QK - 0, QE - 2, QD - 2.5, QC - 3.3, QB - 6.6, QA - $10 \mathrm{mg} / \mathrm{ml}$ of Iscador Qu. 
Table 4. Spermatozoa distance straight line (DSL, $\mu \mathrm{m})$ in experimental groups and time periods.

\begin{tabular}{|c|c|c|c|c|c|}
\hline Group & Mean & S.D. & C.V. & Minimum & Maximum \\
\hline \multicolumn{6}{|l|}{ Time 0} \\
\hline$Q K$ & 17.63 & 3.87 & 21.97 & 10.02 & 26.30 \\
\hline$Q A$ & 16.62 & 3.16 & 19.00 & 12.07 & 23.31 \\
\hline$Q B$ & 18.15 & 3.57 & 15.25 & 11.38 & 24.51 \\
\hline$Q C$ & 19.18 & 4.75 & 20.52 & 8.00 & 26.47 \\
\hline$Q D$ & 17.84 & 3.59 & 20.11 & 9.68 & 24.21 \\
\hline$Q E$ & 16.91 & 3.24 & 19.17 & 11.06 & 23.93 \\
\hline \multicolumn{6}{|l|}{ Time 1} \\
\hline$Q K$ & 22.28 & 3.37 & 15.13 & 16.09 & 29.73 \\
\hline$Q A$ & $12.84^{* * *}$ & 1.52 & 11.88 & 9.67 & 16.74 \\
\hline$Q B$ & $15.33^{* * *}$ & 1.81 & 11.78 & 12.13 & 19.33 \\
\hline$Q C$ & $17.72^{* *}$ & 1.94 & 10.92 & 13.87 & 22.38 \\
\hline$Q D$ & 19.85 & 1.74 & 8.75 & 15.93 & 23.06 \\
\hline$Q E$ & 19.88 & 2.18 & 10.95 & 16.33 & 23.88 \\
\hline \multicolumn{6}{|l|}{ Time 2} \\
\hline$Q K$ & 17.38 & 2.66 & 15.29 & 12.61 & 24.09 \\
\hline$Q A$ & $9.60^{* * *}$ & 2.48 & 25.82 & 0.00 & 13.09 \\
\hline$Q B$ & $12.73^{* * *}$ & 2.04 & 16.05 & 8.87 & 17.83 \\
\hline$Q C$ & $13.53^{* * *}$ & 1.57 & 11.62 & 10.57 & 17.16 \\
\hline$Q D$ & $14.82^{* *}$ & 2.58 & 17.40 & 11.06 & 22.86 \\
\hline$Q E$ & 15.58 & 1.95 & 12.52 & 10.86 & 19.14 \\
\hline \multicolumn{6}{|l|}{ Time 3} \\
\hline$Q K$ & 14.86 & 3.12 & 20.97 & 8.69 & 20.65 \\
\hline$Q A$ & $6.72^{* * *}$ & 2.81 & 41.89 & 0.00 & 10.76 \\
\hline$Q B$ & $10.08^{* * *}$ & 2.75 & 27.26 & 0.00 & 15.48 \\
\hline$Q C$ & $12.12^{* *}$ & 3.16 & 26.10 & 5.58 & 16.52 \\
\hline$Q D$ & 13.31 & 3.37 & 25.33 & 7.81 & 22.37 \\
\hline$Q E$ & 13.60 & 2.12 & 15.59 & 9.66 & 17.87 \\
\hline
\end{tabular}

Legend: S.D. - standard deviation, C.V. - coefficient of variation, *** $(p<0.001), * *(p<0.01)$ (experimental group vs. control). QK -0 , $\mathrm{QE}-2, \mathrm{QD}-2.5, \mathrm{QC}-3.3, \mathrm{QB}-6.6, \mathrm{QA}-10 \mathrm{mg} / \mathrm{ml}$ of Iscador $\mathrm{Qu}$.

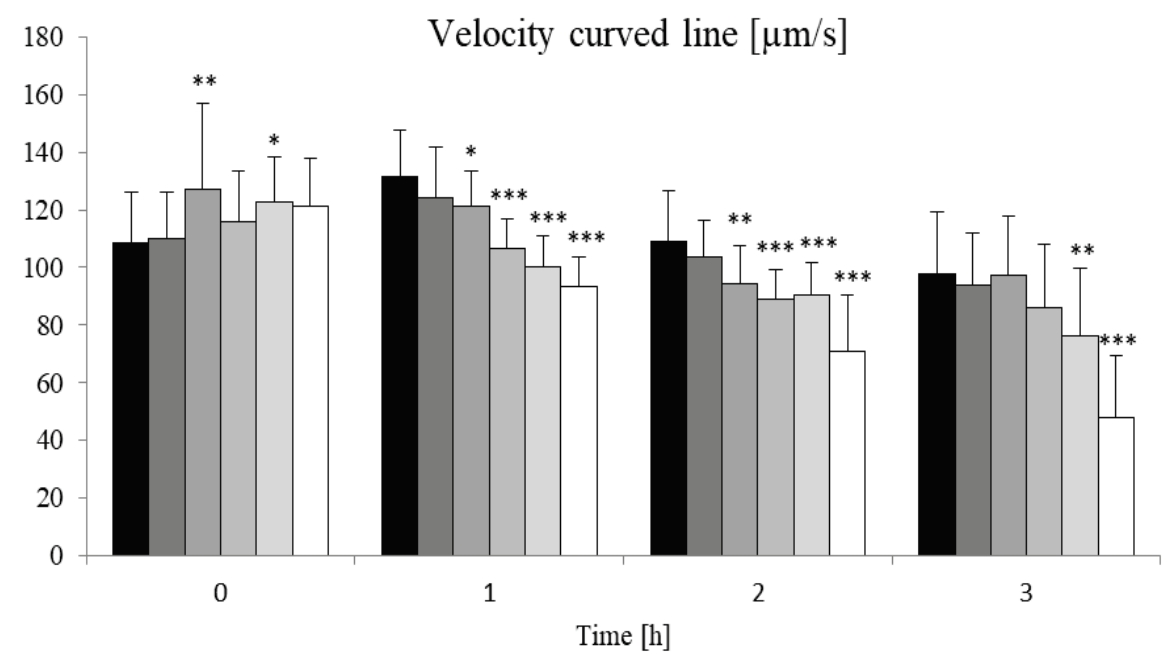

घQK $\square \mathrm{QE} \square \mathrm{QD} \square \mathrm{QC} \square \mathrm{QB} \square \mathrm{QA}$
Fig. 3. The effect of Viscum album quercus on the spermatozoa velocity curved line $(\mu \mathrm{m} / \mathrm{s})$.

$\mathrm{QK}-0, \mathrm{QE}-2, \mathrm{QD}-2.5, \mathrm{QC}-3.3$, $\mathrm{QB}-6.6, \mathrm{QA}-10 \mathrm{mg} / \mathrm{ml}$ of Iscador $\mathrm{Qu}$. The level of significance was set at $* * *(p<0.001), \quad * *(p<0.01) \quad$ and $*(p<0.05)$. 
Table 5. Spermatozoa velocity average path (VAP, $\mu \mathrm{m} / \mathrm{s})$ in experimental groups and time periods.

\begin{tabular}{|c|c|c|c|c|c|}
\hline Group & Mean & S.D. & C.V. & Minimum & Maximum \\
\hline \multicolumn{6}{|l|}{ Time 0} \\
\hline$Q K$ & 52.47 & 10.24 & 19.51 & 33.87 & 73.56 \\
\hline$Q A$ & 57.45 & 9.21 & 16.03 & 39.74 & 73.75 \\
\hline$Q B$ & 60.27 & 9.37 & 15.55 & 43.24 & 79.03 \\
\hline$Q C$ & 59.22 & 12.53 & 21.16 & 27.74 & 76.57 \\
\hline$Q D$ & 60.01 & 12.74 & 21.22 & 30.63 & 82.13 \\
\hline$Q E$ & 53.54 & 9.79 & 18.28 & 37.27 & 73.56 \\
\hline \multicolumn{6}{|l|}{ Time 1} \\
\hline$Q K$ & 68.12 & 8.16 & 11.99 & 50.96 & 86.26 \\
\hline$Q A$ & $41.16^{* * *}$ & 5.22 & 12.68 & 31.48 & 51.78 \\
\hline$Q B$ & $46.62^{* * *}$ & 5.25 & 11.26 & 36.14 & 59.89 \\
\hline$Q C$ & $53.53^{* * *}$ & 6.09 & 11.37 & 43.38 & 64.99 \\
\hline$Q D$ & $60.81^{* * *}$ & 5.87 & 9.65 & 48.00 & 73.53 \\
\hline$Q E$ & $61.79^{* *}$ & 8.34 & 13.50 & 50.35 & 78.56 \\
\hline \multicolumn{6}{|l|}{ Time 2} \\
\hline$Q K$ & 52.56 & 7.84 & 14.91 & 35.50 & 72.26 \\
\hline$Q A$ & $30.32^{* * *}$ & 7.56 & 24.93 & 0.00 & 38.99 \\
\hline$Q B$ & $38.92^{* * *}$ & 5.39 & 13.84 & 29.51 & 49.88 \\
\hline$Q C$ & $40.41^{* * *}$ & 4.07 & 10.07 & 32.51 & 48.94 \\
\hline$Q D$ & $43.58^{* * *}$ & 6.71 & 15.41 & 31.77 & 60.93 \\
\hline$Q E$ & $47.11^{*}$ & 6.80 & 14.43 & 33.09 & 64.36 \\
\hline \multicolumn{6}{|l|}{ Time 3} \\
\hline$Q K$ & 45.30 & 10.31 & 22.75 & 27.50 & 64.75 \\
\hline$Q A$ & $22.61^{* * *}$ & 10.05 & 44.43 & 0.00 & 40.14 \\
\hline$Q B$ & $31.86^{* * *}$ & 9.39 & 29.48 & 0.00 & 49.84 \\
\hline$Q C$ & 38.17 & 10.45 & 27.38 & 19.32 & 52.12 \\
\hline$Q D$ & 42.18 & 10.34 & 24.51 & 25.95 & 61.73 \\
\hline$Q E$ & 41.81 & 7.56 & 18.08 & 28.83 & 52.11 \\
\hline
\end{tabular}

Legend: S.D. - standard deviation, C.V. - coefficient of variation, *** $(p<0.001), * *(p<0.01)$ and $*(p<0.05)$ (experimental group vs. control). QK - 0, QE - 2, QD - 2.5, QC - 3.3, QB - 6.6, QA - $10 \mathrm{mg} / \mathrm{ml}$ of Iscador Qu.

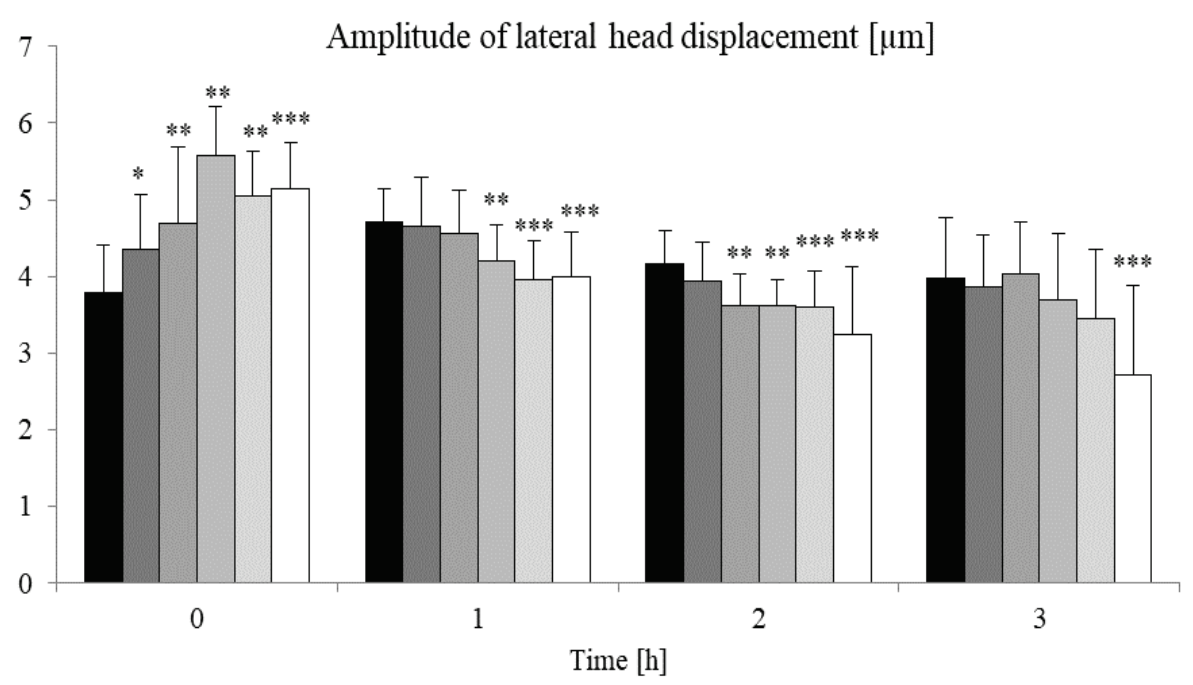

घK $\square \mathrm{QE} \square \mathrm{QD} \square \mathrm{QC} \square \mathrm{QB} \square \mathrm{QA}$
Fig. 4. The effect of Viscum album quercus on the amplitude of lateral head displacement $(\mu \mathrm{m})$.

$\mathrm{QK}$ - 0, QE - 2, QD - 2.5, QC - 3.3, $\mathrm{QB}-6.6, \mathrm{QA}-10 \mathrm{mg} / \mathrm{ml}$ of Iscador $\mathrm{Qu}$. The level of significance was set at $* * *(p<0.001), * *(p<0.01)$ and $*(p<0.05)$. 
Table 6. Spermatozoa velocity straight line (VSL, $\mu \mathrm{m} / \mathrm{s})$ in experimental groups and time periods.

\begin{tabular}{|c|c|c|c|c|c|}
\hline Group & Mean & S.D. & C.V. & Minimum & Maximum \\
\hline \multicolumn{6}{|l|}{ Time 0} \\
\hline$Q K$ & 40.53 & 8.81 & 21.73 & 21.06 & 60.28 \\
\hline$Q A$ & 39.07 & 7.71 & 19.72 & 27.11 & 54.48 \\
\hline$Q B$ & 42.93 & 8.68 & 20.22 & 26.91 & 59.89 \\
\hline$Q C$ & 44.94 & 11.41 & 25.39 & 18.85 & 60.35 \\
\hline$Q D$ & 41.47 & 9.28 & 22.36 & 21.05 & 58.10 \\
\hline$Q E$ & 39.72 & 7.99 & 20.12 & 25.20 & 56.26 \\
\hline \multicolumn{6}{|l|}{ Time 1} \\
\hline$Q K$ & 52.75 & 7.60 & 14.41 & 38.78 & 70.71 \\
\hline$Q A$ & $29.35^{* * *}$ & 3.70 & 12.60 & 21.52 & 37.00 \\
\hline$Q B$ & $35.44^{* * *}$ & 4.17 & 11.78 & 27.66 & 44.88 \\
\hline$Q C$ & $41.50^{* * *}$ & 5.04 & 12.14 & 31.08 & 52.56 \\
\hline$Q D$ & $46.26^{* * *}$ & 4.27 & 9.24 & 36.38 & 55.44 \\
\hline$Q E$ & $46.64^{* * *}$ & 5.25 & 11.25 & 39.21 & 57.29 \\
\hline \multicolumn{6}{|l|}{ Time 2} \\
\hline$Q K$ & 40.72 & 6.28 & 15.41 & 29.37 & 56.76 \\
\hline$Q A$ & $21.99^{* * *}$ & 5.56 & 25.60 & 0.00 & 30.47 \\
\hline$Q B$ & $28.76^{* * *}$ & 4.53 & 15.76 & 20.79 & 40.55 \\
\hline$Q C$ & $31.40^{* * *}$ & 3.55 & 11.31 & 24.63 & 39.57 \\
\hline$Q D$ & $34.09^{* * *}$ & 6.39 & 18.75 & 23.67 & 51.32 \\
\hline$Q E$ & $35.68^{* *}$ & 4.51 & 12.63 & 26.71 & 44.68 \\
\hline \multicolumn{6}{|l|}{ Time 3} \\
\hline$Q K$ & 34.52 & 7.51 & 21.77 & 20.31 & 49.01 \\
\hline$Q A$ & $16.49^{* * *}$ & 7.56 & 45.86 & 0.00 & 31.82 \\
\hline$Q B$ & $22.65^{* * *}$ & 6.40 & 28.23 & 0.00 & 34.89 \\
\hline$Q C$ & $27.81^{* *}$ & 7.45 & 26.80 & 12.09 & 38.72 \\
\hline$Q D$ & 30.22 & 7.86 & 23.01 & 18.52 & 50.15 \\
\hline$Q E$ & 30.80 & 4.97 & 16.14 & 21.21 & 40.57 \\
\hline
\end{tabular}

Legend: S.D. - standard deviation, C.V. - coefficient of variation, $* * *(p<0.001), * *(p<0.01)$ (experimental group vs. control). QK -0 , $\mathrm{QE}-2, \mathrm{QD}-2.5, \mathrm{QC}-3.3, \mathrm{QB}-6.6, \mathrm{QA}-10 \mathrm{mg} / \mathrm{ml}$ of Iscador Qu.

The spermatozoa velocity average path clearly confirm the data for spermatozoa velocity curved line with similar significant decrease (Table 5).

Also the spermatozoa velocity straight line was negative affected by the Viscum album quercus addition. In group with the highest concentration this parameter decreased at Time 1 to $55.64 \%$, at Time 2 to $54.00 \%$ and at Time 3 to $47.77 \%$ compared to control (Table 6 ).

\section{Other fine motility parameters}

The initial amplitude of lateral head displacement (ALH) was $3.78 \pm 0.63 \mu \mathrm{m}$. At Time 0 a statistically significant increase was detected in all experiment groups. After 1 hour of cultivation the ALH was in control group $4.70 \pm 0.44 \mu \mathrm{m}$ and the values decreased by $15.1 \%$ for group QA and $15.9 \%$ for QB. After 2 hours the ALH was the highest in control groups $(4.16 \pm 0.44 \mu \mathrm{m})$ and the most significant decrease was noted in group QA $(3.24 \pm 0.88 \mu \mathrm{m}, \mathrm{p}<0.001)$ with a decrease of $22.1 \%$. After $3 \mathrm{~h}$ of in vitro cultivation, the lowest value was found in the sample QA $(2.72 \pm$ $1.16 \mu \mathrm{m})$ compared to control $(3.98 \pm 0.79 \mu \mathrm{m})$ and was statistically significant (Fig. 4).

The average value of the initial beat cross frequency (BCF) was $29.79 \pm 2.98 \mathrm{~Hz}$. After $1 \mathrm{~h}$ of in vitro cultivation the $\mathrm{BCF}$ in $\mathrm{QK}$ group increased to $31.91 \pm 2.60 \mathrm{~Hz}$. In all experimental samples a statistically significant decreased was found (time- 


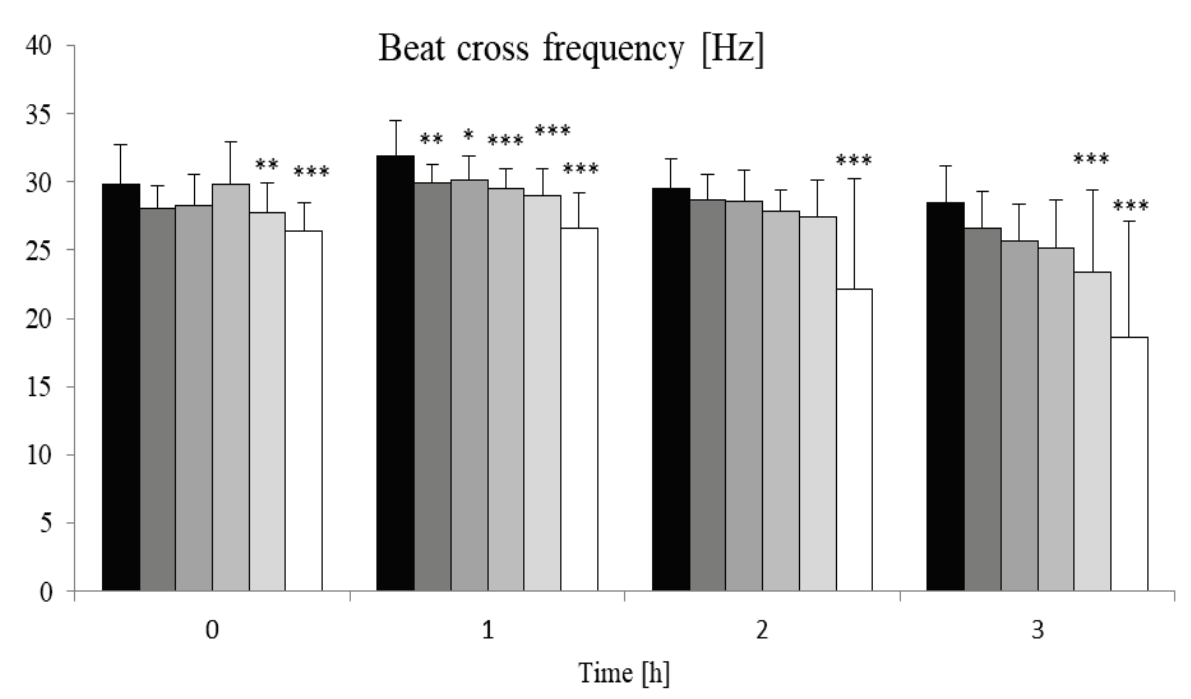

- $\mathrm{QK} \square \mathrm{QE} \square \mathrm{QD} \square \mathrm{QC} \square \mathrm{QB} \square \mathrm{QA}$

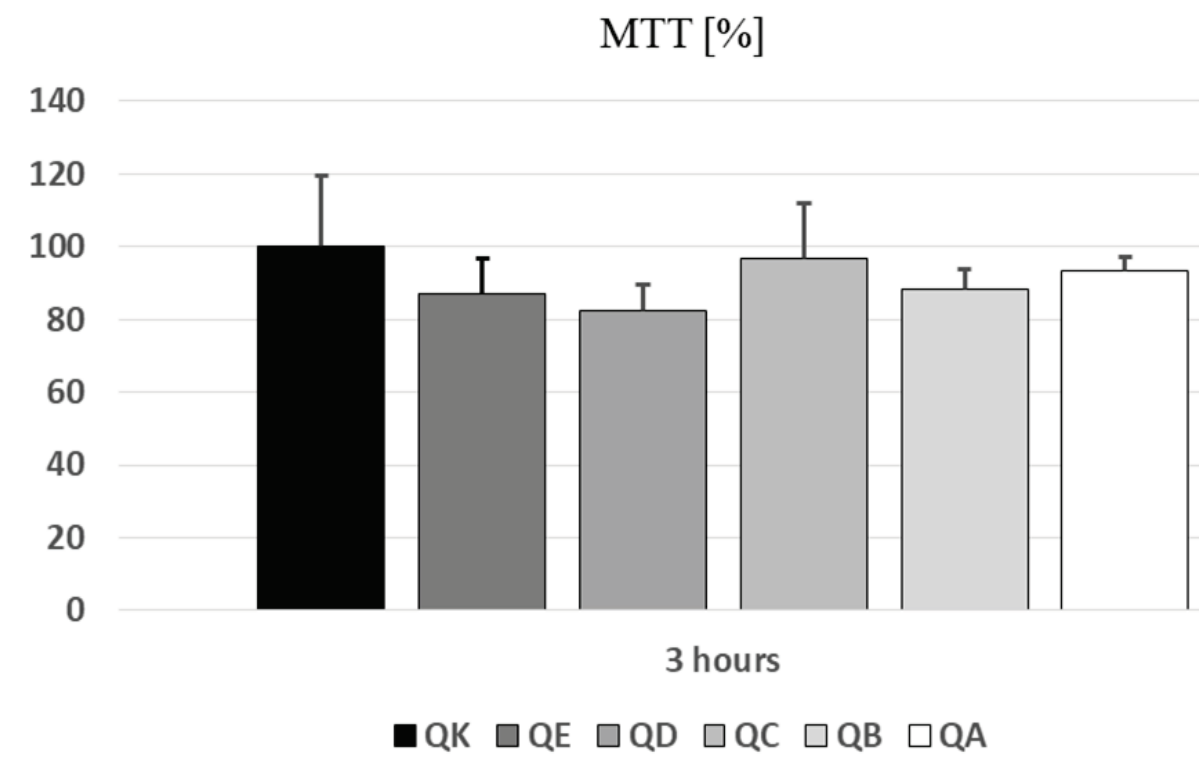

Fig. 5. The effect of Viscum album quercus on the beat cross frequency (Hz). QK - 0, QE - 2, QD - 2.5, QC - 3.3, QB - 6.6, QA - $10 \mathrm{mg} / \mathrm{mL}$ of Iscador Qu. The level of significance was set at $* * *(\mathrm{p}<0.001)$, $* *(p<0.01)$ and $*(p<0.05)$.
Fig. 6. The effect of Viscum album quercus on the viability (\%) of rabbit spermatozoa after 3 hours of incubation.

$\mathrm{QK}$ - 0, QE - 2, QD - 2.5, QC - 3.3, $\mathrm{QB}-6.6, \mathrm{QA}-10 \mathrm{mg} / \mathrm{ml}$ of Iscador $\mathrm{Qu}$. dependent). However, after 2 hours the BCF in control groups (QK) reached 29.55 $\pm 2.18 \mathrm{~Hz}$. The most significant decrease $(25.2 \%)$ was found for group QA $(22.11 \pm 8.10 \mathrm{~Hz})$. After 3 hours the BCF in the control sample was $28.46 \pm 2.75 \mathrm{~Hz}$. In the group QA a statistically significant decrease $(34.6 \%, 18.62 \pm$ $8.49 \mathrm{~Hz})$, and also in the group QB $(17.9 \%, 23.36 \pm 6.03$ $\mathrm{Hz}$ ), compared to the control group was detected (Fig. 5).

The straightness of spermatozoa movement was in all time periods significantly decreased only in group QA (Table 7).

Very similar trends were found for spermatozoa linearity (Table 8).

The spermatozoa wobble was significantly affected only in groups QA and QB (Time 1 and 2) with the highest difference detected at Time $1(0.51 \pm 0.03$ in group QK vs. $0.44 \pm 0.03$ in group QA (Table 9).

Viability, membrane integrity, acrosomal integrity

After 3 hours of incubation, viability of rabbit spermatozoa showed decreased values in all doses of Viscum album quercus in comparison to the control group, but the difference were not significant (Fig. 6).

Significant decrease of membrane integrity (intact) was found in groups with the highest Viscum album quercus concentrations QB (64.00 $2.65 \%$, $\mathrm{p}<0.05)$ and QA $(60.33 \pm 5.51 \%, \mathrm{p}<0.01)$ compared to control group (QK) $(79.33 \pm 1.53 \%)$. Also, in groups $\mathrm{QE}$, QD, QC lower values in comparison to control group were detected $(p<0.05$, Fig. 7). 
Table 7. Spermatozoa straightness (STR) in experimental groups and time periods.

\begin{tabular}{|c|c|c|c|c|c|}
\hline Group & Mean & S.D. & C.V. & Minimum & Maximum \\
\hline \multicolumn{6}{|l|}{ Time 0} \\
\hline$Q K$ & 0.77 & 0.06 & 7.88 & 0.61 & 0.89 \\
\hline$Q A$ & $0.67^{* * *}$ & 0.06 & 9.28 & 0.57 & 0.77 \\
\hline$Q B$ & $0.70^{* *}$ & 0.06 & 9.17 & 0.59 & 0.80 \\
\hline$Q C$ & 0.75 & 0.06 & 8.50 & 0.63 & 0.87 \\
\hline$Q D$ & $0.69^{* * *}$ & 0.11 & 15.98 & 0.45 & 0.84 \\
\hline$Q E$ & 0.73 & 0.04 & 5.16 & 0.67 & 0.82 \\
\hline \multicolumn{6}{|l|}{ Time 1} \\
\hline$Q K$ & 0.77 & 0.04 & 5.57 & 0.62 & 0.86 \\
\hline$Q A$ & $0.71^{* * *}$ & 0.05 & 6.91 & 0.58 & 0.79 \\
\hline$Q B$ & 0.76 & 0.04 & 5.45 & 0.69 & 0.86 \\
\hline$Q C$ & 0.77 & 0.03 & 3.59 & 0.71 & 0.81 \\
\hline$Q D$ & 0.76 & 0.05 & 6.26 & 0.64 & 0.85 \\
\hline$Q E$ & 0.75 & 0.03 & 4.51 & 0.68 & 0.82 \\
\hline \multicolumn{6}{|l|}{ Time 2} \\
\hline$Q K$ & 0.77 & 0.04 & 5.42 & 0.62 & 0.85 \\
\hline$Q A$ & $0.70^{* * *}$ & 0.14 & 19.46 & 0.00 & 0.89 \\
\hline$Q B$ & 0.73 & 0.05 & 6.56 & 0.65 & 0.84 \\
\hline$Q C$ & 0.77 & 0.02 & 3.10 & 0.72 & 0.82 \\
\hline$Q D$ & 0.77 & 0.05 & 5.89 & 0.69 & 0.85 \\
\hline$Q E$ & 0.75 & 0.04 & 5.50 & 0.66 & 0.84 \\
\hline \multicolumn{6}{|l|}{ Time 3} \\
\hline$Q K$ & 0.76 & 0.05 & 6.45 & 0.66 & 0.91 \\
\hline$Q A$ & $0.64^{\mathrm{C}}$ & 0.26 & 40.91 & 0.00 & 31.82 \\
\hline$Q B$ & 0.69 & 0.14 & 20.23 & 0.00 & 0.92 \\
\hline$Q C$ & 0.73 & 0.05 & 6.66 & 0.61 & 0.80 \\
\hline$Q D$ & 0.71 & 0.06 & 8.58 & 0.61 & 0.81 \\
\hline$Q E$ & 0.74 & 0.04 & 5.89 & 0.66 & 0.81 \\
\hline
\end{tabular}

Legend: S.D. - standard deviation, C.V. - coefficient of variation, *** $(p<0.001), * *(p<0.01)$ (experimental group vs. control). QK -0 , $\mathrm{QE}-2, \mathrm{QD}-2.5, \mathrm{QC}-3.3, \mathrm{QB}-6.6, \mathrm{QA}-10 \mathrm{mg} / \mathrm{mL}$ of Iscador Qu.

Membrane integrity - intact [\%]

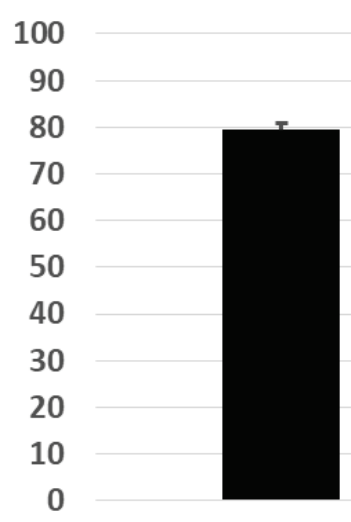

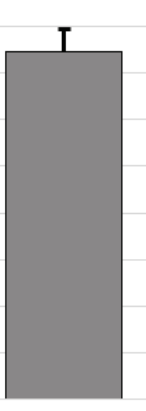

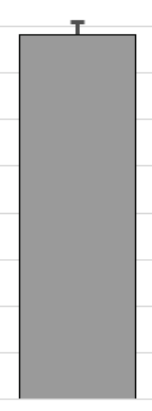



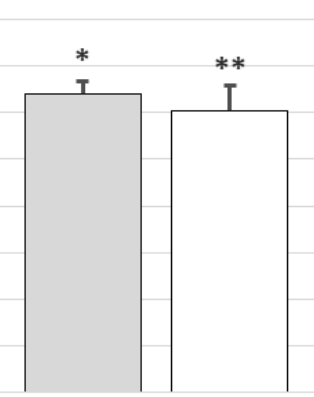

Fig. 7. The effect of Viscum album quercus on the membrane integrity (\%) of rabbit spermatozoa assessed after 3 hours of incubation.

$\mathrm{QK}$ - 0, QE - 2, QD - 2.5, QC - 3.3, QB $6.6, Q A-10 \mathrm{mg} / \mathrm{ml}$ of Iscador $\mathrm{Qu}$. The level of significance was set at $* *(p<0.01)$ and $*(p<0.05)$.

3 hours 
Table 8. Spermatozoa linearity (LIN) in experimental groups and time periods.

\begin{tabular}{|c|c|c|c|c|c|}
\hline Group & Mean & S.D. & C.V. & Minimum & Maximum \\
\hline \multicolumn{6}{|l|}{ Time 0} \\
\hline$Q K$ & 0.37 & 0.05 & 13.31 & 0.23 & 0.45 \\
\hline$Q A$ & $0.32^{* *}$ & 0.03 & 10.81 & 0.26 & 0.37 \\
\hline$Q B$ & 0.34 & 0.05 & 14.89 & 0.27 & 0.44 \\
\hline$Q C$ & 0.38 & 0.07 & 17.39 & 0.24 & 0.48 \\
\hline$Q D$ & 0.33 & 0.09 & 25.70 & 0.18 & 0.47 \\
\hline$Q E$ & 0.35 & 0.04 & 12.00 & 0.27 & 0.42 \\
\hline \multicolumn{6}{|l|}{ Time 1} \\
\hline$Q K$ & 0.40 & 0.04 & 10.28 & 0.29 & 0.50 \\
\hline$Q A$ & $0.31^{* * *}$ & 0.03 & 9.77 & 0.24 & 0.36 \\
\hline$Q B$ & $0.35^{* * *}$ & 0.03 & 8.88 & 0.29 & 0.46 \\
\hline$Q C$ & 0.38 & 0.03 & 7.33 & 0.30 & 0.44 \\
\hline$Q D$ & 0.38 & 0.04 & 9.56 & 0.30 & 0.47 \\
\hline$Q E$ & $0.37^{*}$ & 0.02 & 6.42 & 0.32 & 0.41 \\
\hline \multicolumn{6}{|l|}{ Time 2} \\
\hline$Q K$ & 0.37 & 0.03 & 8.10 & 0.27 & 0.42 \\
\hline$Q A$ & $0.31^{* * *}$ & 0.12 & 38.07 & 0.00 & 0.86 \\
\hline$Q B$ & $0.31^{* * *}$ & 0.03 & 8.75 & 0.26 & 0.38 \\
\hline$Q C$ & 0.35 & 0.03 & 7.42 & 0.30 & 0.40 \\
\hline$Q D$ & 0.35 & 0.04 & 10.76 & 0.28 & 0.42 \\
\hline$Q E$ & 0.34 & 0.02 & 6.99 & 0.29 & 0.39 \\
\hline \multicolumn{6}{|l|}{ Time 3} \\
\hline$Q K$ & 0.35 & 0.03 & 9.49 & 0.30 & 0.44 \\
\hline$Q A$ & 0.32 & 0.18 & 56.85 & 0.00 & 0.74 \\
\hline$Q B$ & 0.29 & 0.11 & 38.09 & 0.00 & 0.81 \\
\hline$Q C$ & 0.32 & 0.04 & 12.17 & 0.24 & 0.40 \\
\hline$Q D$ & 0.30 & 0.04 & 12.96 & 0.25 & 0.39 \\
\hline$Q E$ & 0.33 & 0.03 & 10.36 & 0.27 & 0.40 \\
\hline
\end{tabular}

Legend: S.D. - standard deviation, C.V - coefficient of variation, *** $(p<0.001)$, ** $(p<0.01)$ and $*(p<0.05)$ (experimental group vs. control). QK - 0, QE - 2, QD - 2.5, QC - 3.3, QB - 6.6, QA - $10 \mathrm{mg} / \mathrm{mL}$ of Iscador Qu.

Acrosomal integrity - intact [\%]

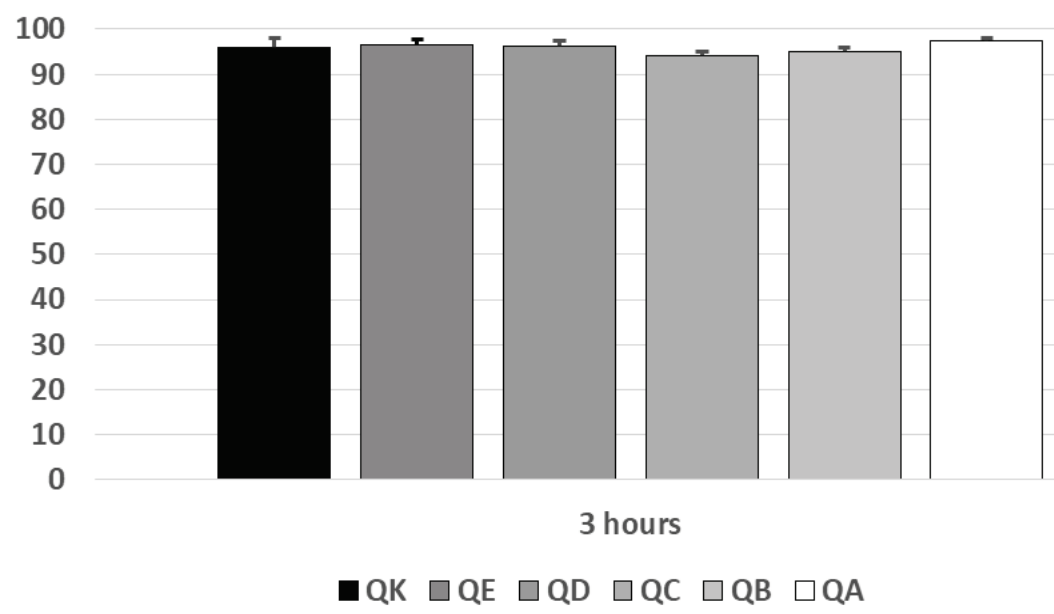

Fig. 8. The effect of Viscum album quercus on the acrosomal integrity (\%) of rabbit spermatozoa assessed after 3 hours of incubation. QK - 0, QE - 2, QD - 2.5, QC $3.3, \mathrm{QB}-6.6, \mathrm{QA}-10 \mathrm{mg} / \mathrm{ml}$ of Iscador Qu. 
Table 9. Spermatozoa wobble (WOB) in experimental groups and time periods

\begin{tabular}{|c|c|c|c|c|c|}
\hline Group & Mean & S.D. & C.V. & Minimum & Maximum \\
\hline \multicolumn{6}{|l|}{ Time 0} \\
\hline$Q K$ & 0.48 & 0.04 & 8.73 & 0.38 & 0.55 \\
\hline$Q A$ & 0.47 & 0.02 & 3.71 & 0.43 & 0.50 \\
\hline$Q B$ & 0.48 & 0.03 & 7.21 & 0.44 & 0.55 \\
\hline$Q C$ & 0.50 & 0.05 & 9.99 & 0.36 & 0.57 \\
\hline$Q D$ & 0.47 & 0.06 & 11.90 & 0.39 & 0.56 \\
\hline$Q E$ & 0.48 & 0.04 & 7.73 & 0.40 & 0.53 \\
\hline \multicolumn{6}{|l|}{ Time 1} \\
\hline$Q K$ & 0.51 & 0.03 & 6.17 & 0.44 & 0.60 \\
\hline$Q A$ & $0.44^{* * *}$ & 0.03 & 6.29 & 0.38 & 0.49 \\
\hline$Q B$ & $0.46^{* * *}$ & 0.02 & 4.89 & 0.42 & 0.53 \\
\hline$Q C$ & 0.50 & 0.03 & 5.35 & 0.43 & 0.56 \\
\hline$Q D$ & 0.50 & 0.02 & 4.84 & 0.46 & 0.57 \\
\hline$Q E$ & $0.49^{*}$ & 0.02 & 4.81 & 0.43 & 0.53 \\
\hline \multicolumn{6}{|l|}{ Time 2} \\
\hline$Q K$ & 0.48 & 0.02 & 4.70 & 0.43 & 0.52 \\
\hline$Q A$ & $0.42^{* *}$ & 0.13 & 30.50 & 0.00 & 0.86 \\
\hline$Q B$ & $0.42^{* *}$ & 0.02 & 5.45 & 0.39 & 0.47 \\
\hline$Q C$ & 0.45 & 0.03 & 6.08 & 0.30 & 0.40 \\
\hline$Q D$ & 0.46 & 0.03 & 6.83 & 0.39 & 0.51 \\
\hline$Q E$ & 0.45 & 0.02 & 4.99 & 0.40 & 0.50 \\
\hline \multicolumn{6}{|l|}{ Time 3} \\
\hline$Q K$ & 0.46 & 0.03 & 6.50 & 0.39 & 0.57 \\
\hline$Q A$ & 0.42 & 0.20 & 46.81 & 0.00 & 0.80 \\
\hline$Q B$ & 0.41 & 0.12 & 28.25 & 0.00 & 0.87 \\
\hline$Q C$ & 0.44 & 0.03 & 7.70 & 0.34 & 0.50 \\
\hline$Q D$ & 0.43 & 0.03 & 6.84 & 0.38 & 0.49 \\
\hline$Q E$ & 0.44 & 0.03 & 6.16 & 0.38 & 0.52 \\
\hline
\end{tabular}

Legend: S.D. - standard deviation, C.V. - coefficient of variation, *** $(p<0.001), * *(p<0.01)$ and $*(p<0.05)$ (experimental group vs. control). QK - 0, QE - 2, QD - 2.5, QC - 3.3, QB - 6.6, QA - $10 \mathrm{mg} / \mathrm{ml}$ of Iscador Qu.

Significant decrease of membrane integrity (intact) was found in groups with the highest Viscum album quercus concentrations QB (64.00 $\pm 2.65 \%$, $\mathrm{p}<0.05)$ and $\mathrm{QA}(60.33 \pm 5.51 \%, \mathrm{p}<0.01)$ compared to control group (QK) $(79.33 \pm 1.53 \%)$. Also, in groups QE, QD, QC lower values in comparison to control group were detected ( $p<0.05$, Fig. 7$)$.

The values of acrosomal integrity showed very similar tendency (94.00-97.33\%) in all experimental groups with no significant difference (Fig. 8).

\section{Discussion}

Researchers are unanimous about the negative impact of various factors on male reproduction and cell/spermatozoa quality (Burton 2013, Lukac et al. 2013,
Petrovova et al. 2014, Lukacova et al. 2015, Kolarova et al. 2017). The mistletoe (Viscum album L.) extract has been shown to be an effective complementary drug in the treatment of cancer patients after surgical removal of the primary tumor. It improved survival, recovery from damage caused by irradiation or cytostatic therapy, and quality of life. In animal tests, clear anti-carcinogenic effects of Iscador were demonstrated. Mainly immune stimulation but also direct cytotoxic activity is believed to be responsible for the anti-carcinogenic activity of Iscador. Tests examining the acute toxicity, genotoxic effects as well as effects on reproduction showed no adverse effects of Iscador preparations. Genotoxic effects and effects on reproduction, which cannot be evaluated in clinical use, have been cleared up in animal tests. Iscador was shown to be clearly non-genotoxic and free of relevant toxic effects 
on reproduction in vivo (Maldacker 2006, Gardin 2009).

On the other hand, it has been stated that anticancer drugs could cause harmful effects on the spermatozoa quality and spermatogenic cell arrangement of male. Therefore, this study was designed to evaluate the in vitro effects of Viscum album quercus (Iscador) on rabbit spermatozoa in order to assess possible beneficial and/or toxic effects of this compound. Rabbits were chosen as the experimental animal in this research for their well-defined reproductive systems (Paal el al. 2014, Rafay and Parkanyi 2016). Some herbal extracts have been proven to have effects on male infertility, for example, gossypol, papaya seed, neem oil, neem seaweed and verbascoside (Mosher and Pratt 1991, Dehghan et al. 2005, Roychoudhury et al. 2009, Okab et al. 2013, Vizzarri et al. 2019).

Since decades, Viscum album preparations have been used in Europe in oncology. They show multifacetted anti-tumor in-vitro activities, which include inhibition of tumor cell proliferation, induction of apoptosis, inhibition of angiogenesis, modulation of immune competence and gene signature expression. Recently, it was demonstrated in vitro that Viscum album exerts an anti-inflammatory effect, mostly directed to chronic inflammation by selectively inhibiting cytokineinduced expression of cyclooxygenase-22 (Seibert et al. 1989, Bussing 2006, Bussing et al. 2008, Hegde et al. 2011, Hajto et al. 2011). Iscador as an anticancer drug used in the treatment of a variety of neoplastic lesions. On the other hand, treatment with Viscum album quercus (Iscador) is accompanied by different toxic effects on different body organs.

An in vitro study investigated the effect of a standardized mistletoe preparation on the action of Trastuzumab, a drug used for the treatment of Her-2 positive breast cancer. A dose dependent antiproliferative effect of Viscum album exctract (VAE) was observed at concentrations $\geq 10 \mu \mathrm{g} / \mathrm{mL}$ after 3 days of incubation. After 7 days a significant growth inhibition of $60 \%$ with the clinically relevant concentration $1 \mu \mathrm{g} / \mathrm{mL}$ was detected and no proliferating cells were left at VAE concentrations of 10 and $100 \mu \mathrm{g} / \mathrm{ml}$. With $10 \mu \mathrm{g} / \mathrm{ml} \mathrm{VAE}$ after 7 days the proportion of early apoptotic cells raised from $9.0 \%$ in the control to $17.4 \%$ and that of late apoptotic/necrotic cells from $18.7 \%$ in control to $78.7 \%$, respectively (Weissenstein et al. 2016).

Effects of Viscum album quercus (VA Qu) extract in various doses were also investigated in an in vitro model with tumor cells: three multiple myeloma
(MM) cell lines (OPM-2, RPMI-8226, U-266) and three B cell lymphoma cell lines (U-698, DOHH-2, WSU-1). Viscum album $/ \mathrm{Qu}$ extract markedly downregulated the membrane expression of IL-6R and gp130 in RPMI-8226 (down to $29 \%$ and $32 \%$ ) and the expression of gp130 in WSU-1 (down to $22 \%$ ). There was a marked reduction of viable cells of RPMI- 8226 (down to $28 \%$ ) and WSU-1 (down to $8 \%$ ) at $100 \mu \mathrm{g} / 10^{6}$ cells $/ \mathrm{ml}$. There was a clear relationship between the inhibition of proliferation and viability. VA Qu was more effective in cells having a high proliferation rate than in those with a low proliferation rate (Kovacs et al. 2006).

Our observations indicate that Iscador in higher doses decreases the spermatozoa motility, progressive spermatozoa motility and some other fine spermatozoa motility parameters (velocity curved line, amplitude of lateral head displacement and beat cross frequency). Thus, Iscador (in high doses in vitro) can have long-term effects on testicular function and can be a potent gonadal toxic drug.

Spermatozoa motility is one of the most important effective factors in male fertility. The mechanism by which Iscador affects the spermatozoa motility has not been clearly elucidated.

A decline in fructose level due to alteration in carbohydrate metabolism after Iscador Qu treatment can be suggested (Rigau et al. 2001, Gren et al. 2011). Reason for decreased spermatozoa motility, can be also a decreased level of androgen carrier proteins involved in spermatozoa motility. Mistletoe lectins have been identified as main active components and exhibit cytotoxic effects. Therefore, probably lectins have a negative effect on the spermatozoa motility found in this study. Also a relationship between diminished spermatozoa quality and anticancer treatment may a result of a series of cascade events that cause a fall in intracellular ATP levels (Luria et al. 2002, Turner 2006, Storey 2008), release of different apoptogenic factors (as pro-caspases, cytochrome $\mathrm{C}$, and apoptosis inducing factor) (Casao et al. 2015) from mitochondria into the cytosol through disruption of mitochondrial membrane, inactivation of some biochemical pathways, enzyme dysfunction, disturbed axonemal protein phosphorylation, increased membrane permeability and generation of spermicidal products, which have adverse effects on the spermatozoa functions. Similarly, the spermatozoa motility is also reduced by ergonovine (Tash and Means 1982, Gallagher and Senger 1989), and the ergot alkaloids induce motility changes in bovine sperm cells 
by interacting with alpha-adrenergic receptors (Wang et al. 2009).

\section{Conclusion}

The results of this study indicate that spermatozoa are a useful in vitro model for the toxicological evaluation of chemicals providing quantitative as well as qualitative data. In conclusion, the present study shows reduction of spermatozoa quality after Viscum album quercus addition in vitro.

\section{Conflict of Interest}

There is no conflict of interest.

\section{Acknowledgments}

This work was supported by projects VEGA 1/0539/18, APVV-16-0289 and KEGA 010/SPU-4/2018.

\section{References}

ADAMKOVICOVA M, TOMAN, R, MARTINIAKOVA M, OMELKA R, BABOSOVA R, KRAJCOVICOVA V, GROSSKOPF B, MASSANYI P: Sperm motility and morphology changes in rats exposed to cadmium and diazinon. Reprod Biol Endocrinol 14: 42, 2016.

BRAEDEL-RUOFF S: Immunomodulatory effects of Viscum album extracts on natural killer cells: Review of clinical trials. Forsch Komplementarmed 17: 63-73, 2010.

BURTON A: Study suggests long-term decline in French sperm quality. Environ Health Perspect 121: a46, 2013.

BUSSING A, BUCKNER U, ENSER-WEIS U: Modulation of chemotherapy-associated immunosuppression by intravenous application of Viscum album L. extract, (Iscador): A randomised phase II study. Eur J Integr Med 1: 2-3, 2008.

BUSSING A: Immune modulation using mistletoe (Viscum album L.) extracts Iscador: Influencing cell function through subcutaneous and intravenous application. Arzneim Forschung 56: 508-515, 2006.

CASAO A, MATA-CAMPUZANO M, ORDAS L, CEBRIAN-PEREZ JA, MUINO-BLANCO T, MARTINEZPASTOR F: Cleaved PARP-1, an apoptotic marker, can be detected in ram spermatozoa. Reprod Domest Anim 50: 688-691, 2015.

CHRENEK P, TRANDZIK J, MASSANYI P, MAKAREVICH A, LUKAC N, PESKOVICOVA D, PALEYANDA R: Effect of transgenesis on reproductive traits of rabbit males. Anim Reprod Sci 99: 127-134, 2007.

COLPI GM, CONTALB GF, NERVA F, SAGONE P, PIEDIFERRO G: Testicular function following chemoradiotherapy. Eur J Obstet Gynecol Reprod Biol 113: 2-6, 2004.

DEHGHAN MH, MARTIN T, DEHGHANAN R: Antifertility effect of Iranian neem seed alcoholic extract on epididymal sperm of mice. Iran J Reprod Med 3: 83-89, 2005.

DI BISCEGLIE C, BERTAGNA A, COMPOSTO ER, LANFRANCO F, BALDI M, MOTTA G, BARBERIS AM, NAPOLITANO E, CASTELLANO E, MANIERI C: Effects of oncological treatments on semen quality in patients with testicular neoplasia or lymphoproliferative disorders. Asian J Androl 15: 425-429, 2013.

FALLAS-LOPEZ M, RODRIGUEZ-DE LARA R, BARCENA-GAMA R, SANCHEZ-TORRES ESQUEDA MT, HERNANDEZ-SANCHEZ D, MARTINEZ-HERNANDEZ PA, AGUILAR-ROMERO O: Rabbit sexual behavior, semen and sperm characteristics when supplemented with sprouted wheat. Anim Reprod Sci 129: 221-228, 2011.

FELENDA JE, TUREK C, STINTZING FC: Antiproliferative potential from aqueous Viscum album L. preparations and their main constituents in comparison with ricin and purothionin on human cancer cells. J Ethnopharmacol 236:100-107, 2019.

GALLAGHER GR, SENGER PL: Effect of phenylephrine, ergonovine, oxytocin and norepinephrine as an extender ingredient on viability of bovine spermatozoa. J Anim Sci 67: 1573-1576, 1989.

GARDIN NE: Immunological response to mistletoe (Viscum album L.) in cancer patients: A four-case series. Phytotherapy Res 23: 407-411, 2009.

GREN A: Effects of Iscador preparations on the reactivity of mouse immune system. Neuro Endocrinol Lett 30: 530-534, 2009. 
GREN A, FORMICKI G, STAWARZ R, MARTINIAKOVA M, OMELKA R: Effect of Iscador on selected parameters of the metabolic block in the animal type Diabetes induced by Alloksan. J Microbiol Biotech Food Sci 1: 215, 2011.

GREN A, FORMICKI G: Effects of iscador and vincristine and 5-fluorouracil on brain, liver, and kidney element levels in alloxan-induced diabetic mice. Biol Trace Elem Res 152: 219-224, 2013.

GREN A, MASSANYI P: Antidiabetic and antioxidant potential of plant extracts. Nitra: Slovak University of Agriculture, Nitra, pp. 125, 2016. ISBN 978-80-552-1524-2

HAJTO T, FODOR K, PERJESI P, NEMETH P: Difficulties and perspectives of immunomodulatory therapy with mistletoe lectins and standardized mistletoe extracts in evidence-based medicine. Evid Based Complement Alternat Med 2011: 1-6, 2011.

HALO M JR, TIRPAK F, DANO A, ZBYNOVSKA K, KOVACIK A, ONDRUSKA L, GREN A, LUKAC N, MASSANYI P. Zinc affects rabbit spermatozoa in vitro: effects on motility and viability. J Microbiol Biotech Food Sci 8: 901-904, 2018.

HEGDE P, MADDUR MS, FRIBOULET A, BAVRY J, KAVERI SV: Viscum album exerts anti-inflammatory effect by selectively inhibiting cytokine-induced expression of cyclooxygenase-2. PLoS One 6: e26312, 2011.

HERÁČEK J, SOBOTKA V, KOLÁTOROVÁ L, KOČÁREK J, HAMPL R: Serum and intratesticular sex steroids in azoospermic men: how do they correlate? Physiol Res 67 (Suppl. 3): S521-S524, 2018.

KOLAROVA H, KRIZOVA J, HULKOVA M, HANSIKOVA H, HULKOVA H, SMID V, ZEMAN J, HONZIK T, TESAROVA M: Changes in transcription pattern lead to a marked decrease in COX, CS and SQR activity after the developmental point of the 22(nd) gestational week. Physiol Res 67: 79-91, 2017.

KOVACS E, LINK S, TOFFOL-SCHMIDT U: Cytostatic and cytocidal effects of mistletoe (Viscum album L.) quercus extract Iscador. Arzneimittelforschung 56: 467-473, 2006.

KROCKOVA J, MASSANYI P, TOMAN R, DANKO J, ROYCHOUDHURY S: In vivo and in vitro effect of bendiocarb on rabbit testicular structure and spermatozoa motility. J Environ Sci Health, Part A 47: 1301-1311, 2012.

LUKAC N, BARDOS L, STAWARZ R, ROYCHOUDHURY S, MAKAREVICH AV, CHRENEK P, DANKO J, MASSANYI P: In vitro effect of nickel on bovine spermatozoa motility and annexin V-labeled membrane changes. J Appl Toxicol 31: 144-149, 2011.

LUKAC N, LUKACOVA J, PINTO B, KNAZICKA Z, TVRDA E, MASSANYI P: The effect of nonylphenol on the motility and viability of bovine spermatozoa in vitro. J Environ Sci Health, Part A 48: 973-979, 2013.

LUKACOVA J, JAMBOR T, KNAZICKA Z, TVRDA E, KOLESAROVA A, LUKAC N: Dose- and time-dependent effects of bisphenol A on bovine spermatozoa in vitro. J Environ Sci Health, Part A 50: 669-676, 2015.

LURIA A, RUBINSTEIN S, LAX Y, BREITBART H: Extracellular adenosine triphosphate stimulates acrosomal exocytosis in bovine spermatozoa via P2 purinoceptor. Biol Reprod 66: 429-437, 2002.

MALDACKER J: Preclinical investigations with mistletoe (Viscum album L.) extract Iscador. Arzneimittelforschung 56: 497-507, 2006.

MALTARIS T, KOELBL H, SEUFERT R, KIESEWETTER F, BECKMANN MW, MUELLER A, DITTRICH R: Gonadal damage and options for fertility preservation in female and male cancer survivors. Asian $J$ Androl 8: 515-533, 2006.

MANGELSDORF I, BUSCHMANN J, ORTHEN B: Some aspects relating to the evaluation of the effects of chemicals on male fertility. Regul Toxicol Pharmacol 37: 356-369, 2003.

MASOPUSTOVA A, JEHLICKA P, HUML M, VOTAVA T, TREFIL L, KRESLOVA M, SYKORA J: Plethysmographic and biochemical markers in the diagnosis of endothelial dysfunction in pediatric acute lymphoblastic leukemia survivors - new applications. Physiol Res 67: 903-909, 2018.

MASSANYI P, CHRENEK P, LUKAC N, MAKAREVICH AV, OSTRO A, ZIVCAK J, BULLA J: Comparison of different evaluation chambers for analysis of rabbit spermatozoa motility using CASA system. Slovak J Anim Sci 41: 60-66, 2008.

MOUSA-BALABEL TM, MOHAMED R A: Effect of different photoperiods and melatonin treatment on rabbit reproductive performance. Vet $Q$ 31: 165-171, 2011. 
MOSHER WD, PRATT WF: Fecundity and infertility in the United States: Incidence and trends. J Fertil Steril 56: 192-193, 1991.

OKAB AB, SAMARA EM, ABDOUN KA, RAFAY J, ONDRUSKA L, PARKANYI V, PIVKO J, AYOUB MA, AL-HAIDARY AA, ALJUMAAH RS, MASSANYI P, LUKAC N: Effects of dietary seaweed (Ulva lactuca) supplementation on the reproductive performance of buck and doe rabbits. J Appl Anim Res 41: 347-355, 2013.

PAAL D, KROCKOVA J, ONDRUSKA L, SLANINA T, STREJCEK F, MASSANYI P: Effect of semen collection frequency on the progress in the motility of rabbit spermatozoa. Slovak J Anim Sci 47: 61-67, 2014.

PARKANYI V, ONDRUSKA L, MASSANYI P, LUKAC N: Effects of the HPA lectin on rabbit sperm motility and fertility. Slovak J Anim Sci 48: 186, 2015.

PETROVOVA E, LUPTAKOVA L, KRESAKOVA L, MALOVESKA M, VDOVIAKOVA K, ELIAS MZJ: Effects of pirimicarb on cell proliferation in immature 3D cell cultures. Folia Veterinaria 58: 169-174, 2014.

POPE CE, ZHANG YZ, DRESSER BL: A simple staining method for quantifying the acrosomal status of cat spermatozoa. Theriogenology 35: 257, 1991.

RAFAY J, PARKANYI V: The rabbit as a model and farm animal at the Research Institute for Animal Production Nitra: A review. Slovak J Anim Sci 49: 141-146, 2016.

RIGAU T, FARRE M, BALLESTER J, MOGAS T, PENA A, RODRIGUEZ-GIL JE: Effects of glucose and fructose on motility patterns of dog spermatozoa from fresh ejaculates. Theriogenology 56: 801-815, 2001.

ROYCHOUDHURY S, MASSANYI P: In vitro copper inhibition of the rabbit spermatozoa motility. $J$ Environ Sci Health, Part A 43: 651-656, 2008.

ROYCHOUDHURY S, MASSANYI P, SLAMECKA J, CHLEBEC I, TRANDZIK J, BULLA J, OKAB AB, TAHA TA, SALEM MH, AYOUB MA: In vitro gossypol induced spermatozoa motility alterations in rabbits. J Environ Sci Health, Part B 44: 730-741, 2009.

ROYCHOUDHURY S, MASSANYI P, BULLA J, CHOUDHURY MD, STRAKA L, LUKAC N, FORMICKI G, DANKOVA M, BARDOS L: In vitro copper toxicity on rabbit spermatozoa motility, morphology and cell membrane integrity. J Environ Sci Health, Part A 45: 1482-1491, 2010.

SAHA R, ROYCHOUDHURY S, KAR K, VARGHESE AC, NANDI P, SHARMA GD, FORMICKI G, SLAMA P, KOLESAROVA A: Coenzyme Q10 ameliorates cadmium induced reproductive toxicity in male rats. Physiol Res 68: 141-145, 2019.

SEIBERT H, KOLOSSA M, WASSERMANN O: Bovine spermatozoa as animal in vitro model for studies on the cytotoxicity of chemicals: Effects of chlorophenols. Cell Biol Toxicol 5: 315-330, 1989.

SLANINA T, KOVACOVA R, LUKAC N, MASSANYI P: Impact of tilmicosin on the rabbit spermatozoa motility and viability. J Microbiol Biotech Food Sci 5: 53-56, 2016.

SLANINA T, MIŠKEJE M, TIRPAK F, BŁASZCZYK M, FORMICKI G, MASSANYI P: Caffeine strongly improves motility parameters of turkey spermatozoa with no effect on cell viability. Acta Vet Hung 66: 137-150, 2018.

STEIN GM, BUSSING A, SCHIETZEL M: Stimulation of the maturation of dendritic cells in vitro by a fermented mistletoe extract. Anticancer Res 22: 4215-4219, 2002.

STOREY BT: Mammalian sperm metabolism: Oxygen and sugar, friend and foe. Int J Dev Biol 52: 427-437, 2008.

SUVEREN E, BAXTER GF, ISKIT AB, TURKER AU: Cardioprotective effects of Viscum album L. subsp. album (European misletoe) leaf extracts in myocardial ischemia and reperfusion. J Ethnopharmacol 209: 203-209, 2017.

TASH JS, MEANS AR: Regulation of protein phosphorylation and motility of sperm by cyclic adenosine monophosphate and calcium. Biol Reprod 26: 745-763, 1982.

TIRPAK F, SLANINA T, KOVACIK A, ONDRUSKA L, MASSANYI P JR, HALO M JR, MASSANYI P: Low taurine concentrations possitively affect rabbit spermatozoa properties in later time intervals. $J$ Microbiol Biotech Food Sci 7: 128-131, 2017.

TVRDA E, LUKAC N, LUKACOVA J, JAMBOR T, MASSANYI P: Dose- and time-dependent in vitro effects of divalent and trivalent iron on the activity of bovine spermatozoa. Biol Trace Elem Res 167: 36-47, 2015.

TVRDA E, MACKOVICH A, GREIFOVA H, HASHIM F, LUKAC N: Antioxidant effects of lycopene on bovine sperm survival and oxidative profile following cryopreservation. Vet Med 62: 429-436, 2017. 
TURNER RM: Moving to the beat: a review of mammalian sperm motility regulation. Reprod Fertil Dev 18: 25-38, 2006.

VIZZARRI F, PALAZZO M, CASAMASSIMA D, ONDRUSKA L, MASSANYI M, TIRPAK F, FORMICKI G, GREN A, MASSANYI P: Lippia citriodora (verbascoside) extract supplementation: Efect on rabbit semen quality in vivo and in vitro. Czech J Anim Sci 64: 1-10, 2019.

WANG H, LOOPER ML, JOHNSON ZB, RORIE RW, ROSENKRANS CF: Involvement of signaling pathways in bovine sperm motility, and effect of ergot alkaloids. In Vitro Cell Dev Biol 45: 483-489, 2009.

WEISSENSTEIN U, KUNZ M, URECH K, REGUEIRO U, BAUMGARTNER S: Interaction of a standardized mistletoe (Viscum album) preparation with antitumor effects of Trastuzumab in vitro. BMC Complement Altern Med 16: 271, 2016.

VITKU J, SOSVOROVA L, CHLUPACOVA T, HAMPL R, HILL M, SOBOTKA V, HERACEK J, BICIKOVA M, STARKA L: Differences in bisphenol A and estrogen levels in the plasma and seminal plasma of men with different degrees of infertility. Physiol Res 64: S303-S311, 2018. 